\title{
Efficient Quantile Regression for Heteroscedastic Models
}

\author{
Yoonsuh Jung \\ Department of Statistics, University of Waikato, Hamilton 3240, New Zealand \\ yoonsuh@waikato.ac.nz \\ Yoonkyung Lee and Steven N. MacEachern \\ Department of Statistics, The Ohio State University, Columbus, Ohio 43210 \\ yklee@stat.osu.edu and snm@stat.osu.edu
}

\begin{abstract}
Quantile regression provides estimates of a range of conditional quantiles. This stands in contrast to traditional regression techniques, which focus on a single conditional mean function. Lee et al. (2012) proposed efficient quantile regression by rounding the sharp corner of the loss. The main modification generally involves an asymmetric $\ell_{2}$ adjustment of the loss function around zero. We extend the idea of $\ell_{2}$ adjusted quantile regression to linear heterogeneous models. The $\ell_{2}$ adjustment is constructed to diminish as sample size grows. Conditions to retain consistency properties are also provided.
\end{abstract}

KEYWORDS: Check loss function; heteroscedasticity; quantile regression

\section{Introduction}

Quantile regression has emerged as a useful tool for providing conditional quantiles of a response variable $Y$ given values of a predictor $X$. This allows us to estimate not only the center, but also the upper or lower tail of the conditional distribution of interest. Due to the ability of quantile regression to capture the full distributional aspects, rather than only the conditional mean, quantile regression has been widely applied. Koenker and Bassett (1978) pioneered quantile regression and proved consistency properties. Bassett and Koenker (1978) showed efficiency of median regression, when the median is more efficient than the mean in a location model. To overcome the restriction of iid errors in linear quantile regression, Gutenbrunner and Jurečková (1992), Koenker and Zhao (1994), and He (1997) developed heterogeneous error models. A comprehensive review is provided in Koenker (2005). 
Quantile regression employs asymmetric absolute loss, the so-called "check loss", as a criterion for minimization. Since the derivative of the check loss does not exist at its minimum, care must be taken with computation. Two main computational strategies exist: linear programming techniques can be used to minimize the criterion function, and hence to solve the problem exactly, (Koenker and D'Orey; 1987; Koenker and Park; 1996), or the cusp of the check loss can be smoothed to allow computational techniques that rely on differentiability. For the latter approach, Horowitz (1998) employed a smooth kernel under median regression, and Nychka et al. (1995) suggested a tiny, symmetric quadratic adjustment of the loss function over the interval $(-\epsilon, \epsilon)$ which is a small enough adjustment to be effectively zero relative to the scale of the data.

Lee et al. (2012) proposed a carefully tailored quadratic adjustment to the loss function that differs in intent from the computational adjustment, and that produces quite different results. Their modification is intended to make quantile regression more efficient, and the modification is asymmetric, except for median regression. The modification leads to superior finite sample performance by exploiting the biasvariance trade-off. To obtain the requisite reduction in variance, the interval of adjustment is not vanishingly small, as are the computationally-motivated adjustments, but is of substantial size. Jung et al. (2010) present a practical rule for choosing the magnitude of adjustment.

Many real applications of regression often call for heterogeneous error models. Although the modified quantile regression in Lee et al. (2012) allows for different local densities around regression quantiles, we expect further efficiency gain in quantile estimation when the differences in the local densities are accounted for. In this paper, we extend the efficient quantile regression method in Lee et al. (2012) to models with heterogeneous error distributions and demonstrate its effectiveness in data analysis. Subsequent sections provide a brief overview of efficient quantile regression, theoretical development for heterogeneous error models, practical strategies for implementation of the method, and documentation of its performance through simulations and data analysis.

\section{Overview of Efficient Quantile Regression}

To estimate the $q$ th regression quantile, the check loss function $\rho_{q}$ is employed:

$$
\rho_{q}(r)=\left\{\begin{array}{cc}
q r & \text { for } r \geq 0 \\
-(1-q) r & \text { for } r<0 .
\end{array}\right.
$$

We first consider a linear model of the form $y_{i}=x_{i}^{\top} \beta+u_{i}$, where $x_{i}, \beta \in \mathbb{R}^{p}$ and the $u_{i}$ 's are iid from some distribution with $q$ th quantile equal to zero. Quantile regression

pursues empirical loss minimization, and so the quantile regression estimator $\hat{\beta}$ is the 
minimizer of

$$
L(\beta)=\sum_{i=1}^{n} \rho_{q}\left(y_{i}-x_{i}^{\top} \beta\right) .
$$

When no covariate is present, this reduces to calculation of the empirical quantile.

As with many estimators driven by piecewise linear loss, the accuracy of quantile estimators can be improved by tapping into the benefits of averaging. Intuitively, averaging the values of observations near the empirical quantile should reduce variance while increasing squared bias only slightly. Motivated by traditional data-analytic methods for handling outliers, Lee et al. (2012) introduce a general framework with inclusion of case-specific parameters to enhance various estimators for robustness or for efficiency. The framework allows formal implementation of the idea of improving the quantile regression estimator with averaging, in particular.

Briefly, the framework in Lee et al. (2012) suggests modifying the quantile regression estimator through the introduction of case-specific parameters $\gamma_{i}$ which change the linear model to $y_{i}=x_{i}^{\top} \beta+\gamma_{i}+u_{i}$. From the fact that this is a super-saturated model, for estimation of $\beta, \gamma=\left(\gamma_{1}, \ldots, \gamma_{n}\right)^{\top}$ should be constrained. Together with the case-specific parameters and an additional penalty for $\gamma$, the objective function to minimize given in (2) is modified to be

$$
L(\beta, \gamma)=\sum_{i=1}^{n} \rho_{q}\left(y_{i}-x_{i}^{\top} \beta-\gamma_{i}\right)+\frac{\lambda_{\gamma}}{2} J(\gamma),
$$

where $J(\gamma)$ is the penalty for $\gamma$ and $\lambda_{\gamma}$ is a penalty parameter. To produce averaging, an $\ell_{2}$ type penalty for the $\gamma$ is considered.

Lee et al. (2012) describe a desired invariance which suggests an asymmetric $\ell_{2}$ penalty of the form $J\left(\gamma_{i}\right) \equiv\{q /(1-q)\} \gamma_{i+}^{2}+\{(1-q) / q\} \gamma_{i-}^{2}$, where $\gamma_{i+}=\max \left(\gamma_{i}, 0\right)$ and $\gamma_{i-}=\max \left(-\gamma_{i}, 0\right)$. For the median, $q=0.5$ and the penalty is a symmetric quadratic. Expression (3) is minimized over $\gamma$ for a fixed $\beta$ as follows. First, note that $\min _{\gamma} L(\hat{\beta}, \gamma)$ decouples to minimization over individual $\gamma_{i}$. Hence, given $\hat{\beta}$ and a residual $r_{i}=y_{i}-x_{i}^{\top} \hat{\beta}, \hat{\gamma}_{i}$ is now defined to be

$$
\arg \min _{\gamma_{i}} \mathcal{L}_{\lambda_{\gamma}}\left(\hat{\beta}, \gamma_{i}\right) \equiv \arg \min _{\gamma_{i}}\left[\rho_{q}\left(r_{i}-\gamma_{i}\right)+\frac{\lambda_{\gamma}}{2} J\left(\gamma_{i}\right)\right],
$$

and is explicitly given by

$$
-\frac{q}{\lambda_{\gamma}} I\left(r_{i}<-\frac{q}{\lambda_{\gamma}}\right)+r_{i} I\left(-\frac{q}{\lambda_{\gamma}} \leq r_{i}<\frac{1-q}{\lambda_{\gamma}}\right)+\frac{1-q}{\lambda_{\gamma}} I\left(r_{i} \geq \frac{1-q}{\lambda_{\gamma}}\right) .
$$

Plugging $\hat{\gamma}$ in (4) produces the $\ell_{2}$ adjusted check loss,

$$
\rho_{q}^{M}(r)=\left\{\begin{array}{ccc}
(q-1) r-\frac{q(1-q)}{2 \lambda_{\gamma}} & \text { for } & r<-\frac{q}{\lambda_{\gamma}} \\
\frac{\lambda_{\gamma}}{2} \frac{1-q}{q} r^{2} & \text { for } & -\frac{q}{\lambda_{\gamma}} \leq r<0 \\
\frac{\lambda_{\gamma}}{2} \frac{q}{1-q} r^{2} & \text { for } & 0 \leq r<\frac{1-q}{\lambda_{\gamma}} \\
q r-\frac{q(1-q)}{2 \lambda_{\gamma}} & \text { for } & r \geq \frac{1-q}{\lambda_{\gamma}} .
\end{array}\right.
$$




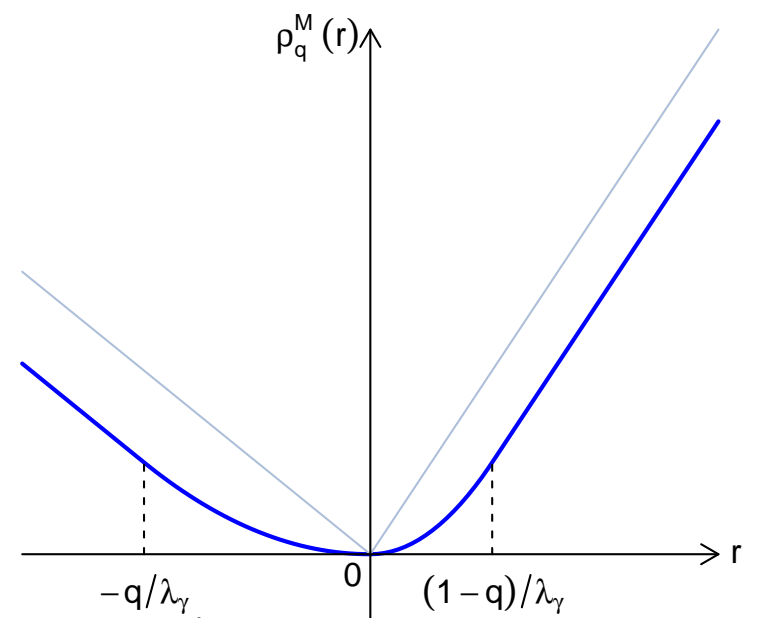

Figure 1: $\ell_{2}$ adjusted check loss in (5) when $q>0.5$.

Figure 1 displays the quadratically adjusted check loss when $q>0.5$. Note that the modified check loss is continuous and differentiable everywhere. The interval of quadratic adjustment is $\left(-q / \lambda_{\gamma},(1-q) / \lambda_{\gamma}\right)$, and we refer to the length of this interval, $1 / \lambda_{\gamma}$, as the "window width". With the loss in (5), we can define the alternative quantile regression estimator $\hat{\beta}^{M}$ as the minimizer of

$$
L_{\lambda_{\gamma}}(\beta)=\sum_{i=1}^{n} \rho_{q}^{M}\left(y_{i}-x_{i}^{\top} \beta\right) .
$$

When the window width of $\rho_{q}^{M}$ is properly chosen, the modified procedure is expected to realize its advantage to the full. For small sample sizes, the window width should be large, capturing a substantial proportion of data in the interval of quadratic adjustment, and it should decay to a width of 0 as $n \rightarrow \infty$ for consistency of the estimator. Jung et al. (2010) (see supplementary materials) provide details of window width selection, recommending use of $\lambda_{\gamma}=c_{q} n^{\alpha} / \hat{\sigma}$, where $c_{q}$ is a constant depending on $q$, $n$ is the sample size, $\alpha$ is a positive constant, and $\hat{\sigma}$ is a robust estimate of the scale of the error distribution. The specification of the window width comes from extensive simulation studies with a wide range of error distributions varying in skewness and tail behaviors and sample sizes. Near-optimal mean squared error (MSE) is obtained across a range of scenarios with $c_{q}=0.5 \exp (-2.118-1.097 \min (q, 1-q))$ and $\alpha=0.3$. As a result, the modified estimator $\hat{\beta}^{M}$ is shown to perform better than the standard quantile regression estimator in terms of MSE in a variety of situations. We henceforth call this estimator the efficient quantile regression estimator, indicating its 
enhanced finite-sample performance.

\section{Efficient Quantile Regression under Heteroge- neous Errors}

The method described in Lee et al. (2012) relies on conditional independence of $y_{i}$ given $x_{i}$, and on correct specification of the quantile function. It does not assume that the $u_{i}$ are identically distributed. As a consequence, the results on consistency and asymptotic normality apply quite broadly. However, one might expect that an estimator that makes use of differences in the distributions of $u_{i}$ would be more efficient. As we show in this section, this is indeed the case.

There are two main approaches to extending quantile regression from the homogeneous error model to the heterogeneous error model. The first approach directly scales the deviations from the quantile surface, so that one considers $\rho_{q}^{M}\left(w_{i}\left(y_{i}-x_{i}^{\top} \beta\right)\right)$ in place of $\rho_{q}^{M}\left(y_{i}-x_{i}^{\top} \beta\right)$, where the values $w_{i}$ are used to produce densities for scaled $u_{i}$ which are locally constant over $i$. This first approach is our primary focus in this work. A second approach plays off the duality between rescaling residuals and attaching weights to cases. This second approach mimics the use of weights in weighted least squares. It also translates into approaches for traditional quantile regression where the linearity of the check loss function allows us to write $\rho_{q}\left(w_{i}\left(y_{i}-x_{i}^{\top} \beta\right)\right)=w_{i} \rho_{q}\left(y_{i}-x_{i}^{\top} \beta\right)$. However, this equality does not hold when $\rho_{q}$ is replaced by $\rho_{q}^{M}$ which combines both linear and quadratic terms. Nevertheless, there is some evidence that this second

approach under $\rho_{q}^{M}$ improves upon quantile regression, as shown in the sequel (also see Jung (2010)). We view this second approach mainly as an ad-hoc improvement on traditional techniques.

In the next two subsections, we first describe the scaled efficient $\mathrm{QR}$ when the scale factors are known, and then consider the case of unknown scales under a location-scale model.

\subsection{Scaled efficient QR with known local densities}

In this subsection, we provide a consistency result for efficient quantile regression based on the modified loss function in (5). We pursue an approach where the scaling occurs inside the loss function, and note the close connection to Koenker (2005). We retain his notation, describing the scale in terms of (its inverse) a weight.

Allowing a potentially different error distribution for each observation, let $Y_{1}, Y_{2}, \ldots$ be independent random variables with $c$ dfs $F_{1}, F_{2}, \ldots$, and suppose that each $F_{i}$ has continuous pdf $f_{i}$. Assume that the $q$ th conditional quantile function of $Y$ given $x$ is linear in $x$ and given by $x^{\top} \beta(q)$, and let $\xi_{i}$ denote the true quantiles $x_{i}^{\top} \beta(q)$. The argument $q$ notes our focus on the $q$ th conditional quantile. When the quantile is clear from context, we omit the argument. First consider the following regularity conditions: 
(C-1) $f_{i}(\xi), i=1,2, \ldots$, are uniformly bounded away from 0 and $\infty$ at $\xi_{i}$.

(C-2) $f_{i}(\xi), i=1,2, \ldots$, admit a first-order Taylor expansion at $\xi_{i}$, and $f_{i}^{\prime}(\xi)$ are uniformly bounded at $\xi_{i}$.

(C-3) There exists a positive definite matrix $D_{0}$ such that $\lim _{n \rightarrow \infty} n^{-1} \sum x_{i} x_{i}^{\top}=D_{0}$.

(C-4) There exists a positive definite matrix $D_{1}$ such that $\lim _{n \rightarrow \infty} n^{-1} \sum f_{i}\left(\xi_{i}\right) x_{i} x_{i}^{\top}=$ $D_{1}$.

(C-5) There exists a positive definite matrix $D_{2}$ such that $\lim _{n \rightarrow \infty} n^{-1} \sum f_{i}^{2}\left(\xi_{i}\right) x_{i} x_{i}^{\top}=$ $D_{2}$.

(C-6) $\max _{i=1, \ldots, n}\left\|x_{i}\right\| / \sqrt{n} \rightarrow 0$.

(C-1), (C-3), (C-4), and (C-6) are the conditions considered for the limiting distribution of the standard regression quantile estimator, and (C-5) for the weighted (or scaled) version of it in Koenker (2005), while (C-2) is an additional assumption that we make.

Under conditions (C-1) through (C-6), Theorem 5.1 in Koenker (2005) states that for $\tilde{\beta}=\arg \min _{\beta \in \mathbb{R}^{p}} \sum_{i=1}^{n} w_{i} \rho_{q}\left(y_{i}-x_{i}^{\top} \beta\right)=\arg \min _{\beta \in \mathbb{R}^{p}} \sum_{i=1}^{n} \rho_{q}\left(w_{i}\left(y_{i}-x_{i}^{\top} \beta\right)\right)$ with $w_{i}=f_{i}\left(\xi_{i}\right)$,

$$
\sqrt{n}(\tilde{\beta}-\beta) \stackrel{d}{\rightarrow} N\left(0, q(1-q) D_{2}^{-1}\right) .
$$

Koenker (2005) proves the result by using the fact that the limiting behavior of

$$
Z_{n}(\delta) \equiv \sum_{i=1}^{n} w_{i}\left\{\rho_{q}\left(u_{i}-x_{i}^{\top} \delta / \sqrt{n}\right)-\rho_{q}\left(u_{i}\right)\right\}=\sum_{i=1}^{n}\left\{\rho_{q}\left(w_{i}\left(u_{i}-x_{i}^{\top} \delta / \sqrt{n}\right)\right)-\rho_{q}\left(w_{i} u_{i}\right)\right\}
$$

determines the limiting distribution of $\tilde{\delta}_{n}=\sqrt{n}(\tilde{\beta}-\beta)$, where $\tilde{\delta}_{n}$ minimizes $Z_{n}(\delta)$ and $u_{i}=y_{i}-x_{i}^{\top} \beta(q)$.

We define the scaled efficient quantile regression estimator as

$$
\tilde{\beta}^{M} \equiv \arg \min _{\beta \in \mathbb{R}^{p}} \sum_{i=1}^{n} \rho_{q}^{M}\left(w_{i}\left(y_{i}-x_{i}^{\top} \beta\right)\right) \text {. }
$$

for any given weights $w_{1}, \ldots, w_{n}$. Similarly, we will consider the limiting behavior of

$$
Z_{n}^{M}(\delta)=\sum_{i=1}^{n}\left\{\rho_{q}^{M}\left(w_{i}\left(u_{i}-x_{i}^{\top} \delta / \sqrt{n}\right)\right)-\rho_{q}\left(w_{i} u_{i}\right)\right\}
$$

To get a consistent estimator, we set the $\lambda_{\gamma}$ in $\rho_{q}^{M}(\cdot)$ to be of the form $c \cdot n^{\alpha}$, where $c$ is a constant, $n$ is the sample size, and $\alpha$ is a positive constant. Under the same condition on $\alpha$ as in the equal weight setting (Lee et al.; 2012), we can show that the scaled quantile regression estimator under $\rho_{q}^{M}$ is consistent and asymptotically normal when the $w_{i}$ 's are proportional to the local densities around the true quantiles $f_{i}\left(\xi_{i}\right)$. 
Theorem 1. Under the conditions (C-1) through (C-6), if $\alpha>1 / 3$ and $w_{i}=f_{i}\left(\xi_{i}\right)$, then

$$
\sqrt{n}\left(\tilde{\beta}^{M}-\beta\right) \stackrel{d}{\rightarrow} N\left(0, q(1-q) D_{2}^{-1}\right) .
$$

The proof of the theorem is in the appendix. Lee et al. (2012) show that the unscaled version of $\hat{\beta}^{M}$ defined in (6) has an asymptotic variance of $q(1-q) D_{1}^{-1} D_{0} D_{1}^{-1}$. To compare $\tilde{\beta}^{M}$ with $\hat{\beta}^{M}$, we examine their asymptotic variances. Let

$$
D=\left(\begin{array}{cc}
D_{2} & D_{1} \\
D_{1} & D_{0}
\end{array}\right)=\lim _{n \rightarrow \infty} n^{-1} \sum_{i=1}^{n}\left(\begin{array}{cc}
f_{i}^{2} & f_{i} \\
f_{i} & 1
\end{array}\right) \otimes x_{i} x_{i}^{\top},
$$

where $\otimes$ represents the Kronecker product. $D$ is a non-negative definite matrix since the matrices in the right hand side of (9) are non-negative definite and the nonnegative definiteness is preserved under the Kronecker product, summation and limit operations. Since all $D_{i}$ in (9) are non-negative definite by (C-3), (C-4), and (C-5), there exists an orthogonal matrix $P$ such that

$$
P^{\top} D P=\left(\begin{array}{cc}
D_{2} & 0 \\
0 & D_{0}-D_{1} D_{2}^{-1} D_{1}
\end{array}\right) .
$$

The fact that $D_{0}-D_{1} D_{2}^{-1} D_{1}$ is non-negative definite and that $D_{1}$ is nonsingular imply that $D_{1}^{-1} D_{0} D_{1}^{-1}-D_{2}^{-1}$ is also non-negative definite. Consequently, the scaled version, $\tilde{\beta}^{M}$, is asymptotically more efficient than $\hat{\beta}^{M}$.

\subsection{Scaled efficient QR with unknown linear scales}

In practice, the true scales of errors are unlikely to be known, and we will be faced with the task of estimating them on the basis of the data. In many circumstances, estimation of the scales will not change the asymptotic properties of the estimators of $\beta$, although we do require a reasonably effective estimator of the scales. In this subsection, we present a result for a location-scale model where both the regression coefficients and the scale of the errors are to be estimated. The model for the $q$ th quantile is

$$
y_{i}=x_{i}^{\top} \beta+\left(x_{i}^{\top} \tau\right) u_{i},
$$

where $u_{i}$ 's are iid from distribution $F_{u}$ with finite density $f_{u}$ such that $F_{u}(0)=q$ and the true quantiles of $Y_{i}$ are $\xi_{i}=x_{i}^{\top} \beta$. The scale for the $i$ th error is given by $x_{i}^{\top} \tau$, leading to the weight $w_{i}=1 /\left(x_{i}^{\top} \tau\right)$. Note that this linear heteroscedastic model is a special case of the heterogeneous error model in the previous section when $f_{i}\left(y_{i}\right)=\frac{1}{\sigma_{i}} f_{u}\left(u_{i}\right)$ with $\sigma_{i} \equiv x_{i}^{\top} \tau>0$. Some of the regularity conditions are slightly modified and restated below for this special case:

(D-5) There exists a positive definite matrix $D_{2}^{*}$ such that $\lim _{n \rightarrow \infty} n^{-1} \sum \frac{x_{i} x_{i}^{\top}}{\left(x_{i}^{\top} \tau\right)^{2}}=D_{2}^{*}$. 
(D-6) $\max _{i=1, \ldots, n}\left\|x_{i} /\left(x_{i}^{\top} \tau\right)\right\|=O\left(n^{1 / 4}\right)$.

(D-6) strengthens (C-6), and it is used to establish a limiting result when $\tau$ is estimated from data to define the linear scales $x_{i}^{\top} \tau$. Koenker and Zhao (1994) define the weighted quantile regression estimator $\check{\beta}_{\tau}$ as $\arg \min _{\beta \in \mathbb{R}^{p}} \sum_{i=1}^{n} \rho_{q}\left(\left(y_{i}-x_{i}^{\top} \beta\right) / x_{i}^{\top} \tau\right)$. With any $\sqrt{n}$-consistent estimator of $\tau$ up to a scale, $\hat{\tau}=\kappa \tau+O_{p}\left(n^{-1 / 2}\right)$, they show that the asymptotic behavior of $\check{\beta}_{\tau}$ is the same as $\check{\beta}_{\hat{\tau}}$, where $\hat{\tau}$ is plugged in. That is,

$\sqrt{n}\left(\check{\beta}_{\hat{\tau}}-\beta\right) \stackrel{d}{\rightarrow} N\left(0, \frac{q(1-q)}{f_{u}^{2}(0)} D_{2}^{*-1}\right)$, which has a smaller asymptotic variance than that of the unweighted version. Note that $\tau$ need only be estimated up to an arbitrary scale factor, as the value of $\kappa$ does not effect the minimization procedure.

Now, with $\rho_{q}^{M}(\cdot)$, the modified quantile regression estimator $\check{\beta}_{\tau}^{M}$ is defined as

$$
\check{\beta}_{\tau}^{M}=\arg \min _{\beta \in \mathbb{R}^{p}} \sum_{i=1}^{n} \rho_{q}^{M}\left(\left(y_{i}-x_{i}^{\top} \beta\right) / x_{i}^{\top} \tau\right) .
$$

With a similar argument as above, a $\sqrt{n}$-consistent estimator of $\tau$ up to a scale will maintain the same consistency properties. Thus, we consider

$$
\check{\beta}_{\hat{\tau}}^{M}=\arg \min _{\beta \in \mathbb{R}^{p}} \sum_{i=1}^{n} \rho_{q}^{M}\left(\left(y_{i}-x_{i}^{\top} \beta\right) / x_{i}^{\top} \hat{\tau}\right) .
$$

Theorem 2. Assume that $x_{i}^{\top} \tau>0$ for all $i$ and that $\hat{\tau}=\kappa \tau+O_{p}\left(n^{-1 / 2}\right)$ for some scalar $\kappa$. Then, under (C-1) through (C-3), (D-5), and (D-6), if $\alpha>1 / 3$, then

$$
\sqrt{n}\left(\check{\beta}_{\hat{\tau}}^{M}-\beta\right) \stackrel{d}{\rightarrow} N\left(0, \frac{q(1-q)}{f_{u}^{2}(0)} D_{2}^{*-1}\right) .
$$

See the appendix for proof. Again, the above estimator is asymptotically more efficient than the unscaled version, and it achieves the same asymptotic efficiency as weighted quantile regression estimates.

The analogy, that weighted least squares is to least squares as weighted quantile regression is to quantile regression, is telling. In addition to motivating both scaled and weighted modified quantile regression, our simulation reveals the type of improvement we expect when we incorporate more features of the model into the analysis. The analogy is farther reaching. If we have approximately correct scales, we expect to see improvement over the unscaled analysis. This suggests the use of a relatively simple model for the scales, perhaps coupled with a more complex model for the quantile function.

\subsection{Estimation of scale factors}

In this section, we outline two practical methods for estimation of the scale factors. Many other strategies are possible and merit future investigation. All such methods 
require some measure that can reveal heteroscedasticity in the data if it is present. With this in mind, and motivated by location-scale models we consider a robust estimate of scale, such as the interquartile range (conditional on $x$ ), $I Q R(x)$. Quantile regression provides a means of estimating this scale function. The fitted $I Q R$ at $i$ th observation is $\widehat{I Q R}_{i}=x_{i}^{\top}(\hat{\beta}(.75)-\hat{\beta}(.25))$, with the $\hat{\beta}(\cdot)$ coming from a pair of quartile regression fits of the data. This allows us to define $\hat{w}_{i}=1 / \widehat{I Q R}_{i}$ and to set $\hat{\tau}=$ $\kappa(\hat{\beta}(.75)-\hat{\beta}(.25))$ for a connection to the theoretical results in the previous subsection. Since $\hat{\beta}(.25)$ and $\hat{\beta}(.75)$ are $\sqrt{n}$-consistent, $\hat{\tau}$ will satisfy the required asymptotic rate in Theorem 2. The positive scalar $\kappa$ is unimportant for asymptotics, but it does impact finite sample performance of the method. We take $\kappa^{-1}=n^{-1} \sum_{i=1}^{n} w_{i}$ to better match the selection of window width described in the supplementary materials. We note that the quantile regression fit used to generate the $\hat{\beta}(q)$ can be replaced with a modified quantile regression fit from $\rho_{q}^{M}(\cdot)$. Our subsequent investigations suggest the effectiveness of this modification. In case the estimated quartiles cross or the IQR estimates are close to zero, we might also consider scale estimates based on more extreme quantiles than the first and the third quartiles. However, as evident in the asymptotic variance of quantile estimators, low probability density at extreme quantiles generally produces larger variance for the corresponding quantile estimates, which will adversely affect the stability of scale estimates.

A second approach focuses on departures of observations from the median regression. Specifically, scale factors can be obtained with the following steps. First, fit a median regression and store the absolute residuals, $\left|r_{i}\right|$. Second, again fit a median regression of $\left|r_{i}\right|$ on $x_{i}$, and obtain a fitted value, $\hat{m}_{i}$, for each case. Finally set the weight to $w_{i}=1 / \hat{m}_{i}$ and proceed to build a scaled quantile regression. In this example, we implicitly set $\hat{\tau}$ to be the estimate of regression coefficient at $q=0.5$ for $\left(x_{i},\left|r_{i}\right|\right)$. The intuitive explanation of this method is that, asymptotically, the median regression surface for $\left|r_{i}\right|$ given $x_{i}$ captures half of the absolute residuals below the surface and half above the surface. If the model is correct, this holds for all $x$. Taking twice this spread gives an interval which (asymptotically) captures half of the probability density at each $x_{i}$. In the case of a symmetric error distribution, $2 \hat{m}_{i}$ provides an estimate of $I Q R_{i}$. The asymptotic properties of this method are more difficult to establish, as the first quantile regressions remove the independence of the $\left|r_{i}\right|$.

These two methods produce a single scale factor for all quantiles for any given vector of covariates. Such methods are appropriate when the errors come from a scale family. When the error distributions depart noticeably from a scale family, one might turn to another method. A natural approach is to attempt to estimate each $f_{i}$ directly, with a method that is local in nature, avoiding the assumption of a location-scale family for the $y_{i}$. While direct estimates of the local density-perhaps kernel density estimates-have great appeal, they also suffer from relatively poor rates of convergence. The theoretical treatment of the previous subsection which echoes results in Koenker and Zhao (1994) suggests the need for a $\sqrt{n}$-consistent estimator.

Our philosophical perspective is to seek a stable estimate of the scale (hence 
the weights) that is reasonably close to consistent, thereby obtaining the bulk of the improvement provided by adjusting for different scales. This pushes us in the direction of relatively robust estimates of the scale and toward approximations such as the location-scale model. It also suggests that we might tweak the estimates in the direction of stability. For example, we might use methods that force separation among the quantiles to prevent the estimated scale from being too close to 0: see He (1997). Or, we might begin with the estimated scales and then shrink them toward uniform scales, yielding a stable procedure which can be considered as midway between unscaled and scaled quantile regression. An example of this hybrid approach is given in Section 4.

Once we have estimated scales in hand, we can apply them to standard quantile regression and modified quantile regression, to produce the corresponding scaled versions. The effectiveness of the scaled procedures is demonstrated in the simulations and real data analyses in the following sections. Our belief is that any appropriate scale estimator that is able to account for the variations in scale will be advantageous. In many settings, the effect of adjusting for scales in some fashion is strong enough that the wide range of procedures we have investigated all show a benefit. The aforementioned methods are but two examples of how to produce estimates of scale factors.

\subsection{Computation}

The modification to the quantile regression estimator in Lee et al. (2012) remains within the framework of M-estimation. The necessary elements for fitting such an estimator include the $\rho$ function (or its derivative, typically called the $\psi$ function) and an overall scaling. For implementation of modified quantile regression, one must write a new $\rho$ (or $\psi$ ) function. Overall scaling in this setting translates to determination of the window width $1 / \lambda_{\gamma}$ which we recommend be set on the basis of the sample size and a preliminary estimate of the dispersion of the errors. General purpose software for M-estimation can be used to fit the models. We have implemented the method in $\mathrm{R}$, making use of the $\mathrm{rlm}$ function in the MASS package along with a function we have written to evaluate the derivative of $(5)$.

The weighted modified quantile regression estimators can be implemented in a similar fashion, provided the weights (or scales) are passed to the M-estimation routine. The weights (or scales) are first estimated with one of the methods in Section 3.3. The $\mathrm{rlm}$ function then allows us to pass weights for M-estimation in one of two ways. Use of the argument wt.method ="inv.var" allows us to create an estimator based on differential scaling with the minimization in (7). Use of the argument wt. method="case" yields minimization of the weighted sum $\sum w_{i} \rho_{q}^{M}\left(y_{i}-x_{i}^{\top} \beta\right)$ over $\beta$. The code used to implement the methods presented in this paper is available from the authors upon request. 


\section{Simulations}

\subsection{Univariate case}

A simple simulation with a heterogeneous error model given below is considered, first with fixed $x$. The heterogeneous error model is given by

$$
y_{i}=x_{i}^{\top} \beta+\left(x_{i}^{\top} \tau\right) \epsilon_{i},
$$

where the $\epsilon_{i}$ 's are iid standard normal, $\beta=\left(\beta_{0}, \beta_{1}\right)^{\top}=(1,2)^{\top}, \tau=(0,1)^{\top}, x_{i}=$ $\left(1, v_{i}\right)^{\top}$, and $v_{i}$ takes one of three values from the set $\{1,2,3\} .2000$ data sets are generated from the model for each of two sample sizes, $n=300$ and $n=900$. In each case, $1 / 3$ of the sample assumes each of the three possible covariate values.

This standard description of the linear model yields the quantile regression model

$$
y_{i}=x_{i}^{\top} \beta(q)+\left(x_{i}^{\top} \tau\right) u_{i}
$$

where $\beta(q)=\left(\beta_{0}, \beta_{1}+\Phi^{-1}(q)\right)^{\top}$ with standard normal cumulative distribution function $\Phi$, and where the $u_{i}$ are iid normals with mean $-\Phi^{-1}(q)$ (hence the qth quantile 0 ) and variance 1 . This description recasts the model in the notation of previous sections.

Five different models are fit to the data; standard quantile regression (QR), weighted QR (WQR), modified quantile regression (QR.M), and two versions of QR.M (WQR.M and WQR.M2) that account for heterogeneous errors. For these versions, WQR.M2 follows the scaling approach and minimizes $\sum_{i=1}^{n} \rho_{q}^{M}\left(w_{i}\left(y_{i}-x_{i}^{\top} \beta\right)\right)$, while WQR.M results from minimization of the weighted sum $\sum_{i=1}^{n} w_{i} \rho_{q}^{M}\left(y_{i}-x_{i}^{\top} \beta\right)$.

The estimate of the weight (or scale) for cases with covariate $x$ is derived from an estimate of the $I Q R$ at $x$. The $I Q R$ can be estimated in a robust fashion, and, for the normal distributions with standard deviation $\sigma$, the $I Q R$ is about $1.35 \sigma$. Specifically, we find the fitted quartile surfaces ( $q=0.75$ and $q=0.25$ ) with unweighted (or unscaled) linear quantile regressions (using either QR or QR.M), and then set $\widehat{I Q R}(x)$ equal to the difference between these two surfaces at $x$. The fitted IQR values from QR are used to derive weights for WQR while those from QR.M are used for WQR.M. The $\widehat{I Q R}_{i}$ are normalized to have mean 1 and we use $\widehat{I Q R}_{i}^{*}=n \cdot \widehat{I Q R}_{i} / \sum_{j=1}^{n} \widehat{I Q R}_{j}$. It is natural to take the scaling approach and minimize $\sum_{i=1}^{n} \rho_{q}^{M}\left(\left(y_{i}-x_{i}^{\top} \beta\right) / \widehat{I Q R}_{i}^{*}\right)$. Alternatively, the value $1 / \widehat{I Q R}_{i}^{*}$ can be used as a weight for the $i$ th observation. Note that these two calculations become equivalent for $\rho_{q}(\cdot)$. In this way, we fit WQR, WQR.M, and WQR.M2 and compare them to their unweighted (or unscaled) counterparts. For all methods, we use $w_{i}=1 / \widehat{I Q R}_{i}^{*}$.

The criterion for evaluation is the mean squared error (MSE) between the true quantile line and each of the five fitted lines for each quantile from $q=0.1$ to 0.5 . For a single replicate, it is estimated by $\operatorname{MSE}(\hat{\beta}(q))=n^{-1} \sum_{i=1}^{n}\left\{x_{i}^{\top} \hat{\beta}(q)-x_{i}^{\top} \beta(q)\right\}^{2}$. Table 1 reveals an interesting pattern in MSE values. The weighted (or scaled) versions 
(WQR, WQR.M, and WQR.M2) perform better than the unweighted (or unscaled) counterparts in every case. Surprisingly, QR.M outperforms WQR when $q \geq 0.3$. In these instances, WQR.M performs even better than QR.M.

Table 1: Point estimate of MSE and standard error of the estimate in parentheses (multiplied by 1000), based on 2000 replicates with $n=300$, and $n=900$, at selected quantiles.

\begin{tabular}{lccccc}
\hline Method & $q=0.1$ & $q=0.2$ & $q=0.3$ & $q=0.4$ & $q=0.5$ \\
\hline QR & $85.71(2.45)$ & $60.64(1.74)$ & $51.75(1.43)$ & $48.44(1.33)$ & $47.03(1.30)$ \\
WQR & $82.91(2.40)$ & $58.12(1.69)$ & $50.27(1.43)$ & $47.64(1.31)$ & $46.08(1.27)$ \\
QR.M & $88.81(2.40)$ & $58.63(1.60)$ & $45.60(1.24)$ & $41.07(1.14)$ & $39.05(1.10)$ \\
WQR.M & $80.11(2.22)$ & $54.61(1.53)$ & $43.65(1.21)$ & $39.75(1.19)$ & $37.98(1.07)$ \\
WQR.M2 & $86.72(2.35)$ & $55.94(1.59)$ & $43.00(1.18)$ & $37.47(1.03)$ & $35.20(0.99)$ \\
\hline QR & $28.56(0.76)$ & $20.15(0.56)$ & $17.32(0.47)$ & $15.61(0.42)$ & $15.50(0.41)$ \\
WQR & $27.39(0.75)$ & $19.47(0.55)$ & $16.97(0.46)$ & $15.22(0.41)$ & $15.18(0.41)$ \\
QR.M & $28.85(0.74)$ & $19.33(0.54)$ & $15.84(0.43)$ & $13.87(0.37)$ & $13.19(0.36)$ \\
WQR.M & $27.49(0.71)$ & $18.25(0.51)$ & $15.25(0.42)$ & $13.54(0.37)$ & $12.95(0.35)$ \\
WQR.M2 & $28.43(0.80)$ & $18.62(0.54)$ & $15.01(0.42)$ & $12.95(0.35)$ & $12.06(0.32)$ \\
\hline
\end{tabular}

In many data analyses, the covariates are best viewed as arising at random. Our second simulation examines this case. We generate the $x_{i}$ independently from the uniform distribution over the interval $(1,3)$.

Under the heterogeneous model mentioned above, 2000 data sets are simulated with a sample size of 300. Standard normal and exponential with mean one error distributions are considered for $\epsilon_{i}$ in (12). Again, the MSE is employed to measure the accuracy of the five fitted models. Table 2 summarizes the simulation results and illustrates that all of the MSEs for the weighted (or scaled) methods (WQR, WQR.M, and WQR.M2) are smaller than their counterparts (QR, QR.M), by 3-5\% on average. The MSE for WQR.M and WQR.M2 is less than that for WQR, except for $q=0.1$, under the normal distribution. This assessment demonstrates a clear benefit from use of the weights or scales in the modified quantile regression. To quantify the benefit of WQR.M and WQR.M2 in efficiency relative to WQR, we define the efficiency gain as the fraction of reduction in MSE by using WQR.M and WQR.M2 relative to the MSE of WQR. Table 3 shows the size of efficiency gain for WQR.M and WQR.M2 using the MSE values in Table 2. Efficiency gains range mostly from $10 \%$ to $20 \%$ except for $q=0.1$ for the normal and $q=0.5$ for the exponential distribution. While there is little difference between the two error distributions in terms of the range, the direction of increase in efficiency gain differs. For both distributions, the size of efficiency gain appears to be bigger for the quantiles with high density. 
Table 2: Point estimate of MSE and standard error of the estimate in parentheses (multiplied by 1000), based on 2000 replicates with $n=300$ under standard normal and Exponential(1) error distributions at selected quantiles.

\begin{tabular}{lccccc}
\hline Method & $q=0.1$ & $q=0.2$ & $q=0.3$ & $q=0.4$ & $q=0.5$ \\
\hline & & $\mathrm{N}(0,1)$ \\
QR & $77.70(2.05)$ & $55.19(1.42)$ & $46.53(1.17)$ & $44.57(1.09)$ & $43.74(1.08)$ \\
WQR & $75.89(1.93)$ & $54.07(1.39)$ & $45.08(1.16)$ & $42.62(1.04)$ & $41.91(1.04)$ \\
QR.M & $79.49(1.95)$ & $50.55(1.26)$ & $40.67(1.00)$ & $36.67(0.91)$ & $34.96(0.85)$ \\
WQR.M & $73.57(1.81)$ & $47.55(1.20)$ & $38.71(0.97)$ & $35.11(0.87)$ & $33.62(0.83)$ \\
WQR.M2 & $79.23(2.01)$ & $49.33(1.28)$ & $38.49(0.97)$ & $34.00(0.84)$ & $32.29(0.79)$ \\
\hline & & $\operatorname{Exp(1)}$ \\
QR & $3.26(0.09)$ & $6.80(0.19)$ & $11.34(0.30)$ & $17.70(0.49)$ & $27.34(0.72)$ \\
WQR & $3.14(0.09)$ & $6.52(0.18)$ & $10.96(0.28)$ & $17.06(0.46)$ & $26.36(0.70)$ \\
QR.M & $2.60(0.08)$ & $5.69(0.16)$ & $9.74(0.27)$ & $15.79(0.43)$ & $26.87(0.71)$ \\
WQR.M & $2.46(0.08)$ & $5.37(0.15)$ & $9.17(0.26)$ & $14.72(0.40)$ & $24.35(0.64)$ \\
WQR.M2 & $2.42(0.08)$ & $5.36(0.15)$ & $9.23(0.26)$ & $15.22(0.43)$ & $26.70(0.75)$ \\
\hline
\end{tabular}

Table 3: Efficiency gain of WQR.M and WQR.M2 relative to WQR based on MSE values in Table 2.

\begin{tabular}{|c|c|c|c|c|c|c|c|c|c|c|}
\hline \multirow[b]{2}{*}{$q$} & \multicolumn{5}{|c|}{$\mathrm{N}(0,1)$} & \multicolumn{5}{|c|}{$\operatorname{Exp}(1)$} \\
\hline & 0.1 & 0.2 & 0.3 & 0.4 & 0.5 & 0.1 & $\overline{0.2}$ & 0.3 & 0.4 & 0.5 \\
\hline WQR.M & 0.03 & 0.12 & 0.14 & 0.18 & 0.20 & 0.22 & 0.18 & 0.16 & 0.14 & 0.08 \\
\hline WQR.M2 & -0.04 & 0.09 & 0.15 & 0.20 & 0.23 & 0.23 & 0.18 & 0.16 & 0.11 & -0.01 \\
\hline
\end{tabular}

\subsection{Multivariate case}

Many uses of quantile regression involve more than one covariate and the data often show various features such as outliers, thick tails for the residuals, or cases with unusually high leverage. We have created a set of simulations to investigate these issues. Some of the results are presented in this section, with additional results available as supplementary materials.

All of the simulations involve a linear quantile regression model. We begin with a standard linear model of the form

$$
y_{i}=x_{i}^{\top} \beta+\left(x_{i}^{\top} \tau\right) \epsilon_{i},
$$

where the $\epsilon_{i}$ are errors coming from a distribution $F$ with median 0 . This translates to the quantile regression model:

$$
y_{i}=x_{i}^{\top} \beta(q)+\left(x_{i}^{\top} \tau\right) u_{i},
$$


where $u_{i}$ has $q$ th quantile 0 and $\beta(q)=\beta+\tau F^{-1}(q)$. In all settings, the model includes an intercept and eight covariates, and the sample size is $n=100$. The $\tau$ vector drives the differences in scale: $\tau=(20,2,2,0,0,0,0,4,4)^{\top}$. We have used $\beta=$ $(10,3,1.5,0,0,2,0,0,0)^{\top}$. As in the univariate simulation study, we evaluate MSE of the estimators from the five methods for the quantiles at $q=(0.1,0.25,0.5,0.8)$. The results we report are based on 2000 replicates.

Throughout this set of simulations, we obtain scales/weights by the following procedure. First, we obtain normalized $\widehat{I Q R}_{i}^{*}$ as described in Section 4.1. Second, these estimates are shrunk toward uniform scales with the calculation $\widehat{I Q R}_{i, \alpha}^{*}=$ $\alpha \cdot \widehat{I Q R}_{i}^{*}+(1-\alpha)$. Note that the average of $\widehat{I Q R}_{i}^{*}$ is 1 . The parameter $\alpha$ determines the amount of shrinkage toward uniformity. $\alpha=0$ corresponds to an unweighted analysis and $\alpha=1$ to no shrinkage at all. Our investigations have found that stabilizing the estimated weights through shrinkage improves performance of the estimators. This is true for both modified and unmodified quantile regression. As a general rule, less shrinkage is needed for larger sample sizes. More shrinkage is needed when the optimal scales (i.e., true $I Q R_{i} \mathrm{~s}$ ) vary less. Where needed, weights are defined to be $w_{i}=1 / \widehat{I Q R}_{i, \alpha}^{*}$. The results reported in this paper use $\alpha=0.5$. The supplementary materials contain tables of results for $\alpha=0,0.1,0.3$, and 0.5 .

We investigate four cases. The first case is a traditional normal-theory regression setting where $F$ is a standard normal distribution and the $\epsilon_{i}$ are iid. As there are eight covariates, $x_{i}$ consists of a 1 followed by a vector drawn from the truncated multivariate normal distribution with mean vector 0 and variance $\Sigma=\left[\rho^{|i-j|}\right]$ with $\rho=0.5$. The truncation enforces $x_{i}^{\top} \tau>0$. The covariate vectors are independent for $i=1, \ldots, 100$, and they are independent of the $\epsilon_{i}$. The results are shown in upper left panel of Figure 2. The two best performing methods are WQR.M and WQR.M2. Use of the weights reduces MSE by roughly $10 \%$. This reduction is in addition to the reduction obtained from modifying the check loss function which produced reductions in MSE of $2 \%$ to $17 \%$.

The second case focuses on outliers. A preliminary set of $\epsilon_{i}$ is generated as in case $1.5 \%$ of the errors are then chosen at random and tripled. Thus, marginally, $\epsilon_{i}$ comes from a mixture of two mean 0 normal distributions, having variances 1 and 9. Covariates are generated as in case 1. As our focus is on the robustness of the procedures, we use $\beta(q)=\beta+\tau \Phi^{-1}(q)$ for evaluation of MSE, corresponding to the quantile regression surface without the outliers. The upper right panel of Figure 2 shows this evaluation. For all but the most extreme quantile $(q=0.1)$, WQR.M and WQR.M2 are the best performers. For quantiles in the range 0.25 to 0.8 , the reduction in MSE due to inclusion of weights/scales ranges from $9 \%$ to $15 \%$. Again, this is in addition to the reduction that follows from modifying the check loss function. WQR has the smallest MSE for $q=0.1$, although with $5 \%$ contamination, we have less interest in performance at the extreme quantiles.

The third case examines a thick tailed error distribution. The data are generated as in case 2, but the target for evaluation now comes from the mixture distribution 


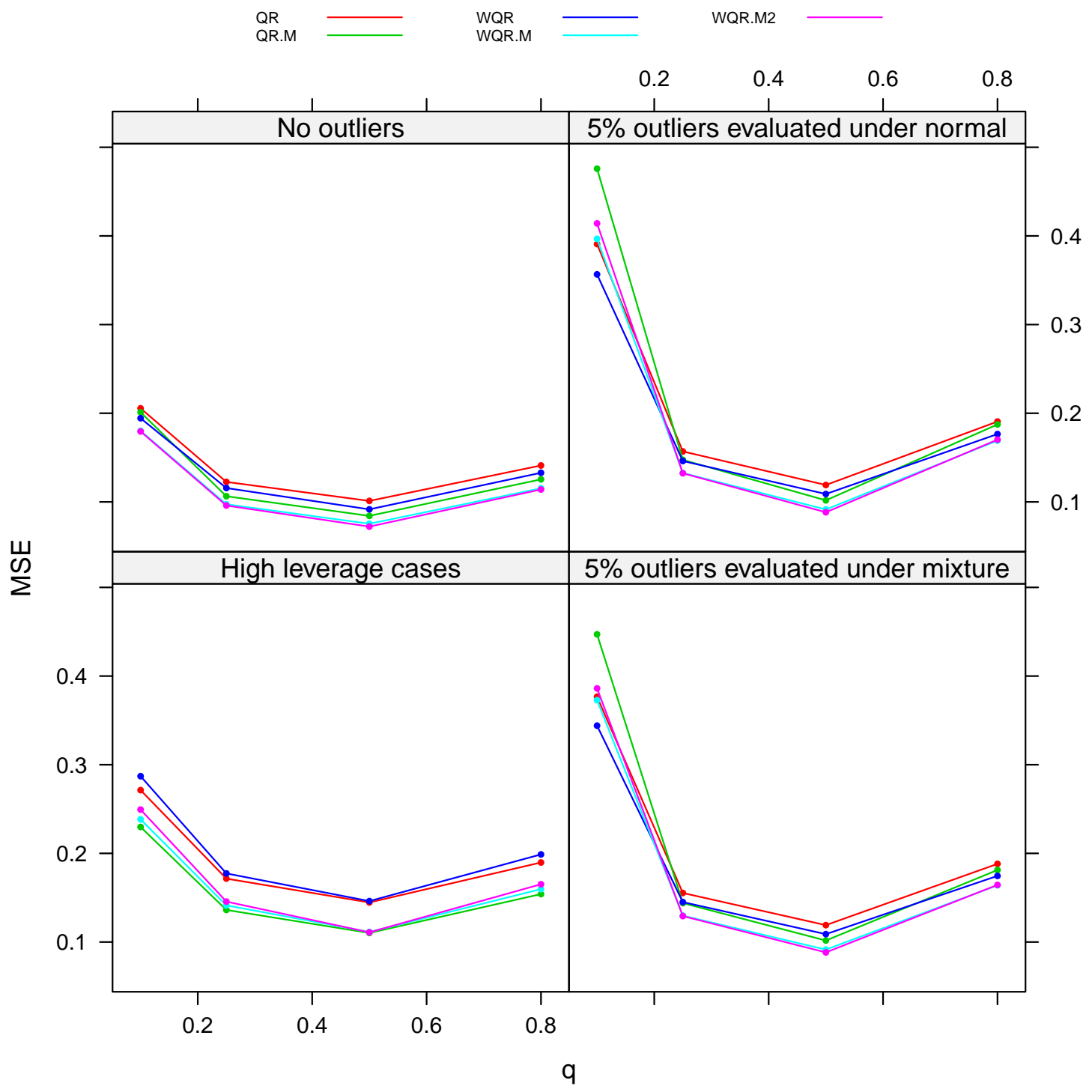

Figure 2: Each panel shows MSE for QR, WQR, QR.M, WQR.M, and WQR.M2 for the quantiles $q=(0.1,0.25,0.5$, and 0.8$)$. The upper left panel is for normal errors. The upper right panel is for normal errors with $5 \%$ outliers mixed in, evaluated under the normal target. The lower right panel is for normal errors with $5 \%$ outliers mixed in, evaluated under the mixture distribution target. The lower left panel has normal errors, but with $3 \%$ of cases having high leverage. 
itself, so that $\beta(q)=\beta+\tau F^{-1}(q)$, with $F$ a mixture of a standard normal distribution (weight 0.95 ) and a $N(0,9)$ distribution (weight 0.05 ). The lower right panel of Figure 2 shows this evaluation. Again, for quantiles in the range 0.25 to 0.8 , the best performers are WQR.M and WQR.M2. Reductions in MSE due to inclusion of the weights/scales ranges from $9 \%$ to $15 \%$ on top of the reduction from modifying the check loss. WQR is the best performer for $q=0.1$, with WQR.M and WQR.M2 trailing by $8 \%$ and $12 \%$, respectively.

The fourth case investigates the impact of high leverage on the methods. The $\epsilon_{i}$ are generated as in case 1 . The covariate vectors are initially generated as in case 1 . The leverage of each case is computed as for an ordinary least squares fit to the data. For the $3 \%$ of cases with the highest leverages, the covariates are altered. The covariates are either all doubled or they are all multiplied by negative 2. For a given case, the alteration which yields the greater variance is used (this ensures that the variance will be positive). The lower left panel of Figure 2 shows this evaluation. The best method is QR.M, followed by WQR.M and WQR.M2. There is an increase in MSE due to use of the weights/scales of $1 \%$ to $9 \%$. All of the modified methods outperform both of the traditional methods. We note that performance of the weighted/scaled methods in this case is improved by shrinking the estimated interquartile ranges more strongly toward uniformity.

Taken as a whole, the simulations show the value of using weights for quantile regression. The improvement due to inclusion of weight/scale appears to be slightly greater for modified quantile regression than for traditional quantile regression. The simulations also show the enhanced finite-sample efficiency of modified quantile regression. Further improvement is possible by tailoring the amount of shrinkage to the problem at hand.

\section{Empirical Examples}

\subsection{GDP growth data}

In this section, we apply the heterogeneous error model developed in Section 3 to an economic growth data set. The data consist of 161 observations on determinants of cross-country gross domestic product (GDP) growth rates and were used in Koenker and Machado (1999). The first 71 observations are on the period 1965-75, the remainder on 1975-85. There are 13 covariates and one response variable, "Annual Change Per Capita GDP". The response variable indicates the rate of annual GDP growth with most of the observed values between -0.05 and 0.05 . Our analysis focuses on the relationship between one of the covariates, "\% of Female High School Graduate or More" and the response variable. The five methods (QR, WQR, QR.M, WQR.M, and WQR.M2) are applied to the data, and the weights/scales are estimated using $\mathrm{IQR}$ as in Section 4.1. Before analyzing the data, we removed two extreme outliers because their inclusion resulted in negative weights under $\mathrm{QR}$, as the fitted first quar- 
tile exceeded the fitted third quartile within the range of the data. There was no such problem with QR.M, reaffirming a pattern observed elsewhere, that QR.M reduces crossings of the fitted quantile surfaces.

The panels in Figure 3 show the fitted lines for the quantiles 0.05, 0.1, 0.25, 0.5, 0.75, and 0.9 from the four methods. First, QR and WQR produce quite different fits, especially for the lower and upper quantiles, while QR.M and WQR.M2 yield similar fits. We conjecture that this difference arises from the poor fit of $\mathrm{QR}$ (this view is supported by the simulation study). The $\widehat{I Q R}$ or the fitted weights for individual cases provide clearer evidence for this conjecture. The rightmost observation in Figure 3 has the largest weight for both WQR and WQR.M2. If the weights are normalized to sum to one, the weight for this observation from QR.M is only 0.0265 , approximately four times the mean weight of $1 / 159 \approx 0.0063$. However, the QR fit produces a weight of 0.4883 for this observation, which is about 78 times the mean weight and almost two hundred times larger than the smallest weight. We believe that the more moderate swing in weights is preferable for this data set, and so prefer the weights generated by QR.M to those generated by QR.

The eventual fits of the models under WQR and WQR.M2 show only minor differences for small values of the covariate, but big differences for large values of the covariate. The estimated differences between the 10th and 90th percentiles when no females graduate from high school are 0.075927 and 0.075280 , while the estimated difference when $50 \%$ of females have graduated from high school are 0.002497 and 0.025519. We find the modest spread estimated under the WQR.M2 method for large values of the covariate to be far more plausible than the near-degeneracy of $80 \%$ of the distribution estimated under the WQR method.

To compare QR.M, WQR.M and WQR.M2 in terms of prediction accuracy, we used cross validation. Given the modest size of the data set, we used 1000 repetitions of 5 -fold cross validation. For each repetition, the data were randomly partitioned into five sets (or folds), each of size 32 or 33 . The model was fit to four of the folds with the fifth fold reserved for validation. This was repeated with each fold serving as the validation data. To create the cross validation (CV) score, we used the defining loss function for a quantile, the check loss function. The CV score is given by $\operatorname{CV}(\hat{\beta}(q))=n^{-1} \sum_{i=1}^{n} \rho_{q}\left(y_{i}-x_{i}^{\top} \hat{\beta}^{[-k(i)]}(q)\right)$, where $k(i)$ indexes the fold containing observation $i$ and $\hat{\beta}^{[-k]}(q)$ indicates the estimate of $\beta(q)$ from the data, excluding the $k$ th fold. We examined a number of quantiles with $q=0.1,0.25,0.5,0.75$, and 0.9 . The mean discrepancy between the fitted and observed values appears as a single dot in Figure 4 with pre-specified $q$, giving us 5000 points in each panel. The figure conveys a clear advantage for the weighted method with the bulk of the points falling below the 45 degree line, with the lone exception of $q=0.25$. A numerical summary of the cross validation score (CV score) appears in Table 4. In a similar way, WQR.M2 is also compared to QR.M in Figure 5. Except for the extreme quantiles of $q=0.1$ and 0.9, WQR.M2 produces smaller CV scores than QR.M.

As explained in Section 4, weights (or scales) are estimated from the fitted $I Q R$ 
Table 4: Point estimate of mean cross validated score (CV score) and standard error of the estimate in parentheses (multiplied by 10,000), based on 1000 different splits of "Annual Change Per Capita GDP" data. CV score is measured by check loss function.

\begin{tabular}{lccccc}
\hline Method & $q=0.1$ & $q=0.25$ & $q=0.5$ & $q=0.75$ & $q=0.9$ \\
\hline QR & $7.147(0.0678)$ & $5.959(0.0948)$ & $6.082(0.0621)$ & $8.298(0.1024)$ & $7.801(0.0970)$ \\
WQR & $7.189(0.0674)$ & $6.034(0.1009)$ & $5.204(0.0598)$ & $5.347(0.0902)$ & $7.946(0.0942)$ \\
QR.M & $6.901(0.0535)$ & $5.403(0.0574)$ & $5.567(0.0484)$ & $5.193(0.0523)$ & $3.626(0.0519)$ \\
WQR.M & $6.689(0.0545)$ & $5.647(0.0582)$ & $4.847(0.0491)$ & $4.812(0.0536)$ & $3.429(0.0521)$ \\
WQR.M2 & $6.911(0.0482)$ & $5.207(0.0569)$ & $4.599(0.0479)$ & $3.989(0.0468)$ & $3.515(0.0504)$ \\
\hline
\end{tabular}

with unweighted QR or QR.M for fitting the weighted (or scaled) counterparts at $q$ $=(0.25,0.5,0.75)$. For the more extreme quantiles $q=0.1$ and 0.9 , we have used the reciprocal of the fitted distance between $q=0.9$ and 0.1 in place of the fitted $I Q R$. The weights or scales are then used for WQR, WQR.M and WQR.M2 as before. Estimating the weights or scales with a range that extends beyond the upper and lower quartiles generally improves the fits, (equivalently reduces the CV score) at extreme quantiles such as $q=0.1$ and 0.9 .

\subsection{Corrected Boston Housing data}

The Boston Housing data set was originally examined by Rubinfeld (1978) to detect social and environmental factors that affect house prices in the Boston Metropolitan area. The data set is composed of 506 census tracts (observations) with 14 variables where median house value is considered as the response variable. Gilley and Pace (1996) provide details of corrections made to some of the data. The response variable and 13 explanatory variables are $C M E D V$ (corrected median values of owner-occupied housing in USD 1000), CRIM (crimes per capita), ZN (proportion of residential land zoned for lots over 25,000 sqft), INDUS (proportion of non-retail business acres per town), CHAS (a factor with levels 1 if tract borders Charles River; 0 otherwise), NOX (nitric oxides concentration in parts per 10 million), $R M$ (average number of rooms per dwelling), AGE (proportion of owner-occupied units built prior to 1940), DIS (weighted distance to five Boston employment centers), $R A D$ (index of accessibility to radial highways), TAX (full-value property-tax rate per USD 10,000), PTRATIO (pupil-teacher ratio), $B\left(1000(A A-0.63)^{2}\right.$ where $A A$ is the proportion of African American), and $L S T A T$ (percentage values of lower status population).

To begin the analysis, we explored the data set by fitting weighted and unweighted quantile regression models. This led us in the same direction as Rubinfeld (1978), and we transformed a number of variables for the subsequent analysis. We used 

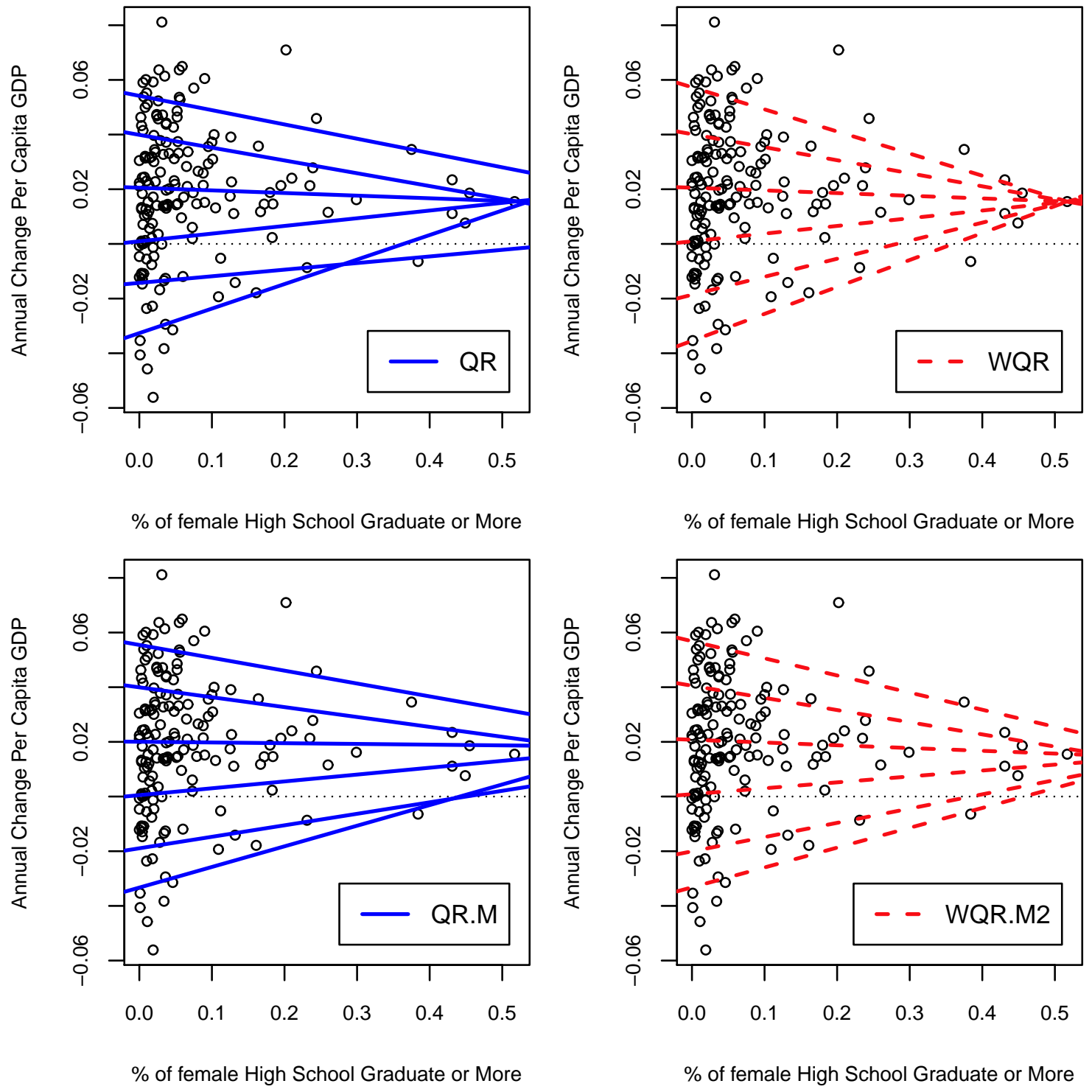

Figure 3: The panels show quantile regression lines estimated by QR and WQR (top) and by QR.M and WQR.M2 (bottom) at $q=(0.05,0.1,0.25,0.5,0.75$, and 0.9). 


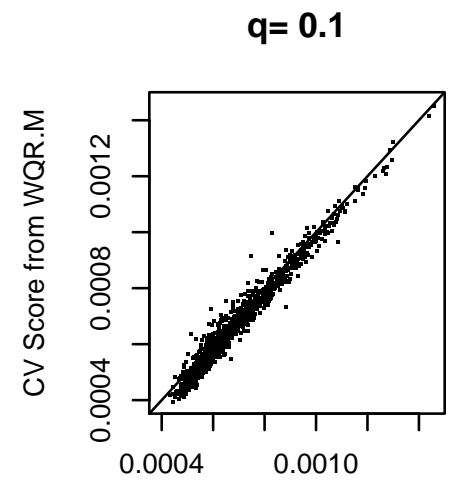

CV Score from QR.M

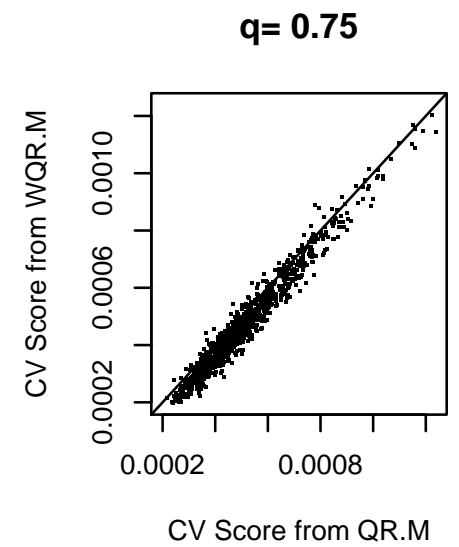

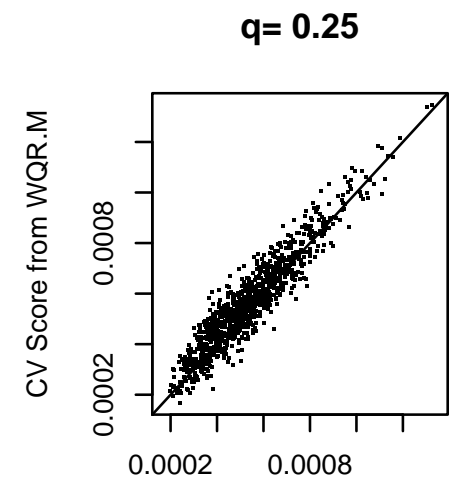

CV Score from QR.M

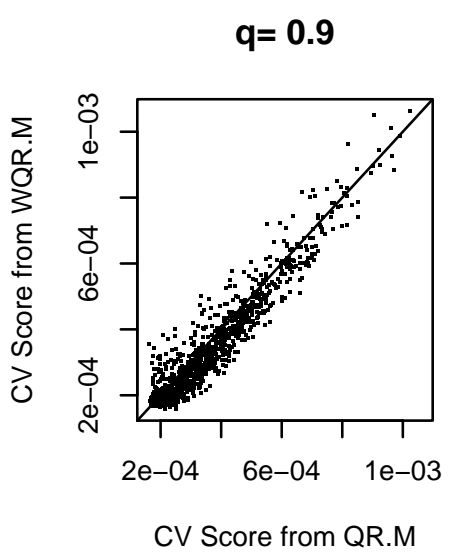

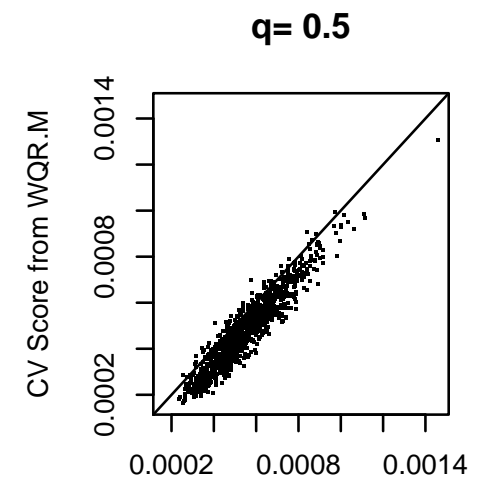

CV Score from QR.M

Figure 4: CV scores by QR.M and WQR.M at $q=(0.1,0.25,0.5,0.75$, and 0.9$)$ with a line of slope 1 from "Annual Change Per Capita GDP" data. Weights are estimated from $I Q R$ at $q=(0.25,0.5,0.75)$, while the distance between 0.9 and 0.1 fitted lines is used to estimate the weights at $q=(0.1,0.9)$. 

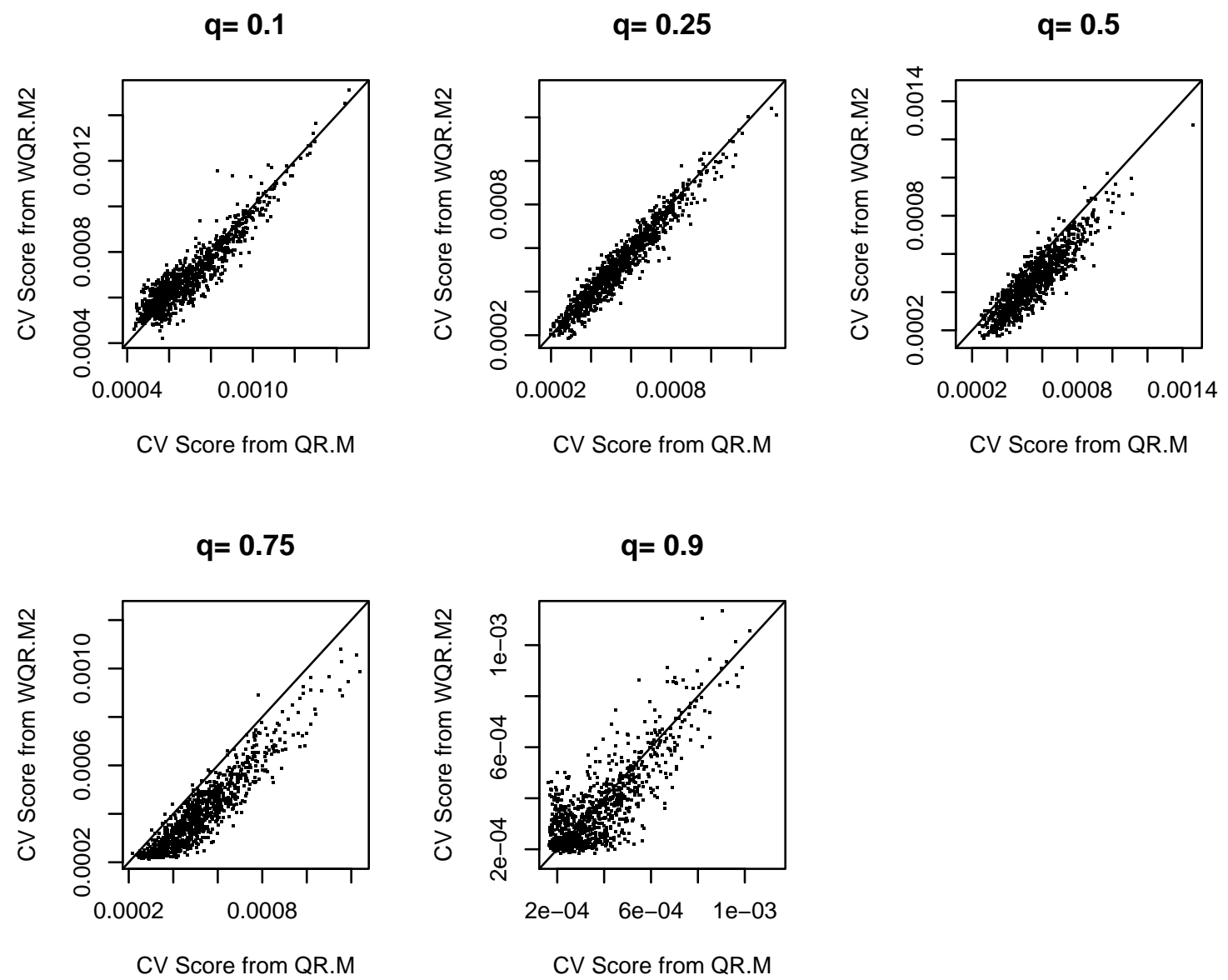

Figure 5: CV scores by QR.M and WQR.M2 at $q=(0.1,0.25,0.5,0.75$, and 0.9$)$ with a line of slope 1 from "Annual Change Per Capita GDP" data. Weights are estimated from $I Q R$ at $q=(0.25,0.5,0.75)$, while the distance between 0.9 and 0.1 fitted lines is used to estimate the weights at $q=(0.1,0.9)$. 
$\log (C M E D V), \log (D I S), \log (R A D), \log (L S T A T), N O X^{2}$, and $R M^{2}$. Since $Z N$ and INDUS are rarely significant at various quantiles, they were excluded and one gigantic outlier was removed before the data analysis. In shorthand notation, the final model we build is,

$$
\begin{aligned}
\log (C M E D V)= & C R I M+C H A S+N O X^{2}+R M^{2}+A G E+\log (D I S)+ \\
& \log (R A D)+T A X+P T R A T I O+B+\log (\text { LSTAT }) .
\end{aligned}
$$

The model is fit with five procedures, QR, WQR, QR.M, WQR.M, and WQR.M2, for $q$ ranging from 0.1 to 0.9 . Weights (or scales) are estimated in two steps. First, a standard median regression (say, $\eta\left(x_{i}\right)=\operatorname{med}\left(y_{i} \mid x_{i}\right)$ ) is fit, and the fits $\left(\hat{\eta}_{i}=\hat{\eta}\left(x_{i}\right)\right.$ ) and residuals $\left(r_{i}=y_{i}-\hat{\eta}_{i}\right)$ are obtained. Second comes a single-predictor median regression treating the fit as the independent variable and the absolute value of the residual as the response (say, $m\left(x_{i}\right)=\operatorname{med}\left(\left|r_{i}\right| \mid \hat{\eta}_{i}\right)$ ). Use of the fit as a single predictor stabilizes the second regression, leading to more stable weights (and scales). The weights are taken to be proportional to the reciprocals of the fitted values from the second regression $\left(w_{i} \propto 1 / \hat{m}\left(x_{i}\right)\right)$.

To compare the performance of the five methods of fitting the model, prediction accuracy is examined through 5-fold cross validation as in Section 5.1. A summary of 500 different partitions of the data is given in Table 5 .

Table 5: Point estimate of mean cross validated score (CV score) and standard error of the estimate in parenthesis (multiplied by 10,000), based on 500 different splits of "Corrected Boston Housing" data. CV score is measured by check loss function.

\begin{tabular}{lcccccc}
\hline Method & $q=0.05$ & $q=0.1$ & $q=0.25$ & $q=0.5$ & $q=0.7$ & $q=0.95$ \\
\hline QR & $187.8(0.25)$ & $304.2(0.22)$ & $537.5(0.33)$ & $717.5(0.45)$ & $699.4(0.55)$ & $374.1(0.68)$ \\
WQR & $186.6(0.22)$ & $304.0(0.24)$ & $538.2(0.33)$ & $715.9(0.46)$ & $697.8(0.54)$ & $373.5(0.69)$ \\
QR.M & $187.2(0.23)$ & $303.5(0.22)$ & $535.5(0.33)$ & $714.2(0.45)$ & $694.3(0.53)$ & $370.8(0.65)$ \\
WQR.M & $185.7(0.21)$ & $303.5(0.23)$ & $535.2(0.31)$ & $712.1(0.42)$ & $695.7(0.52)$ & $369.4(0.67)$ \\
WQR.M2 & $185.2(0.21)$ & $302.6(0.22)$ & $536.0(0.32)$ & $714.0(0.43)$ & $695.1(0.52)$ & $370.4(0.67)$ \\
\hline
\end{tabular}

In overall terms, the weighted and scaled versions perform better than their counterparts, although there are some exceptions. These exceptions appear to be driven by a departure of our implicit model for the scale of the error distribution. The actual data show a departure from the linear relationship we have used for the scale, and so a more flexible model for weights and scales is desirable. Additionally, the appropriate local scale seems to depend on $q$, the quantile under examination. This suggests the need for a more refined method for determining the weights and scales. We do not pursue such a method here, but do indicate directions to explore in the discussion section. In any event, even with misspecification of the weights (or scales), weighting (and scaling) produces an overall net benefit. 
A second feature of Table 5 is even more striking. The fits from the $l_{2}$ adjusted check loss (QR.M and WQR.M) show uniformly smaller CV score values than that from the original check loss (QR and WQR, respectively). Modification of the check loss to enhance efficiency is undoubtedly worthwhile.

To further investigate the effect of weights and scales on the fit of the model, the estimated coefficients from the four methods are drawn in Figure 6 for a range of quantiles from 0.1 to 0.9 . The (transformed) covariates are standardized before fitting the above model so that the coefficients are comparable. Tables containing the coefficients fit under the various methods are available in the supplementary materials. The coefficients under the various fits truly differ. Figure 7 directly shows these differences which, in some cases, amount to approximately half of the coefficient. Some of the more noticeable differences appear for $R M^{2}$ and $\log (L S T A T)$. The differences in these coefficients appear in the figure as the two lines toward the top of the first two panels. Substantial differences are also apparent for $A G E$ and $\log (R A D)$ which appear toward the bottom of the figure. The differences are largest for extreme quantiles of house price. The third panel of Figure 7 shows the differences between WQR and WQR.M2. These differences are smaller in magnitude with no clear pattern emerging.

Synthesizing the information in the figures and tables, we see similar effects on the quantile regressions when we include weights for both traditional quantile regression (QR) and modified quantile regression (QR.M). This suggests that the effect is not an artifact driven by details of a particular estimation technique. Instead, we attribute it to the heteroscedasticity which is present in the data. Thus, we see the need to account for heteroscedasticity in the quantile regression. The improvement due to modification is most apparent in Table 5, where modification leads to uniformly improved performance on a measure (CV score) which would naturally favor the unmodified methods. Thus, we see the benefits that follow from modification of the check loss function. Together, we have need of both modification and weighting/scaling.

\section{Discussion}

We have proposed a new quantile regression method for analyzing heteroscedastic data, which extends the methodology developed in Lee et al. (2012) to allow for heterogeneity in the error distributions. Asymptotic results establish the large-sample behavior of the method, placing it on a firm theoretical footing. Details of implementation have been considered, and a practical method to implement the technique has been proposed. The success of the method relative to standard quantile regression has been shown through simulation studies and an examination of two data sets. We attribute the success of WQR.M and WQR.M2 relative to WQR partly to improved estimation of weights (and scales) with QR.M instead of QR and partly to the modification of the check loss function. There are many variations on the general theme 


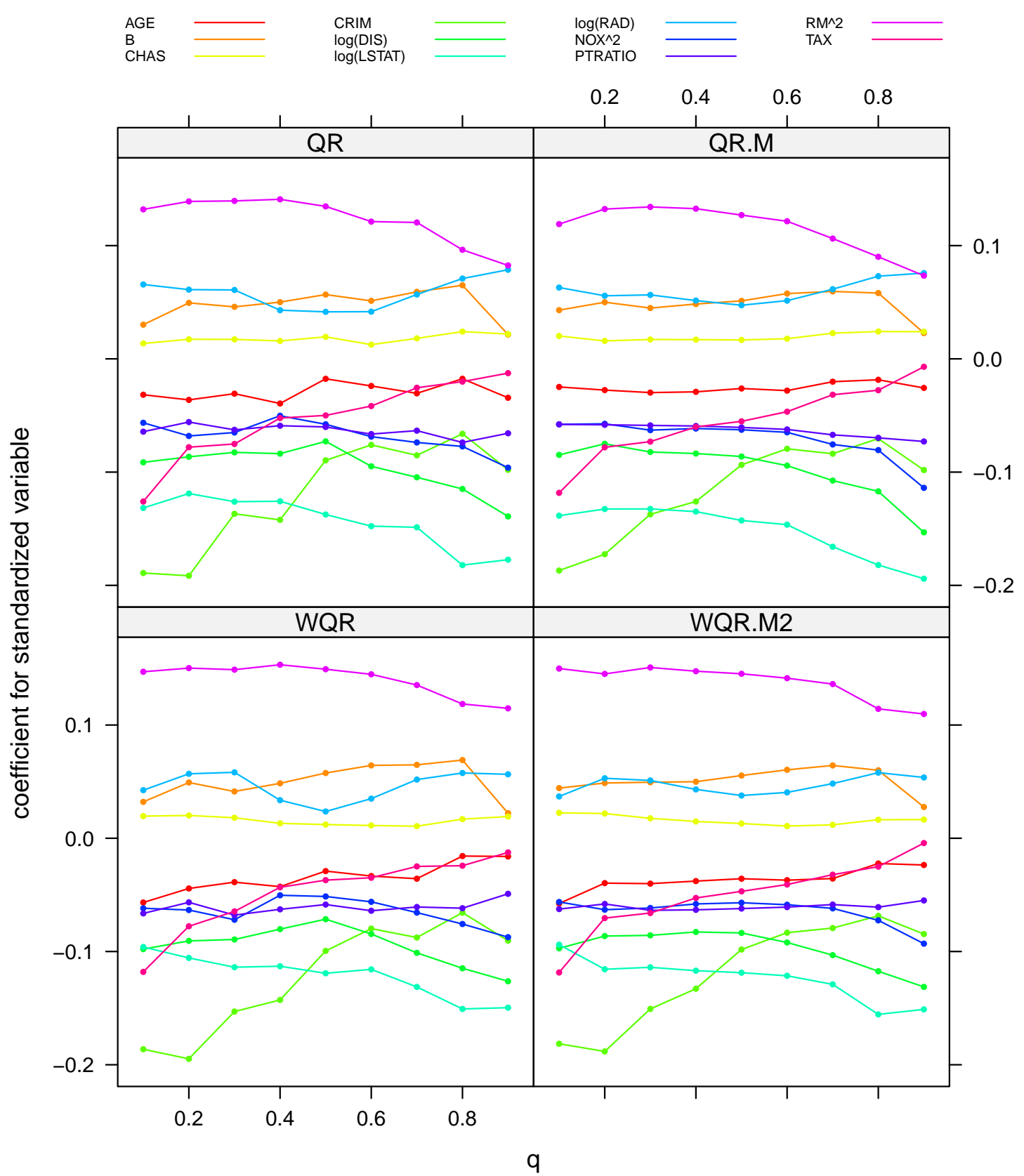

Figure 6: Estimated coefficients of the standardized covariates for Boston Housing data at various quantiles $(q=0.1, \ldots, 0.9)$ using QR, WQR, QR.M and WQR.M2. 


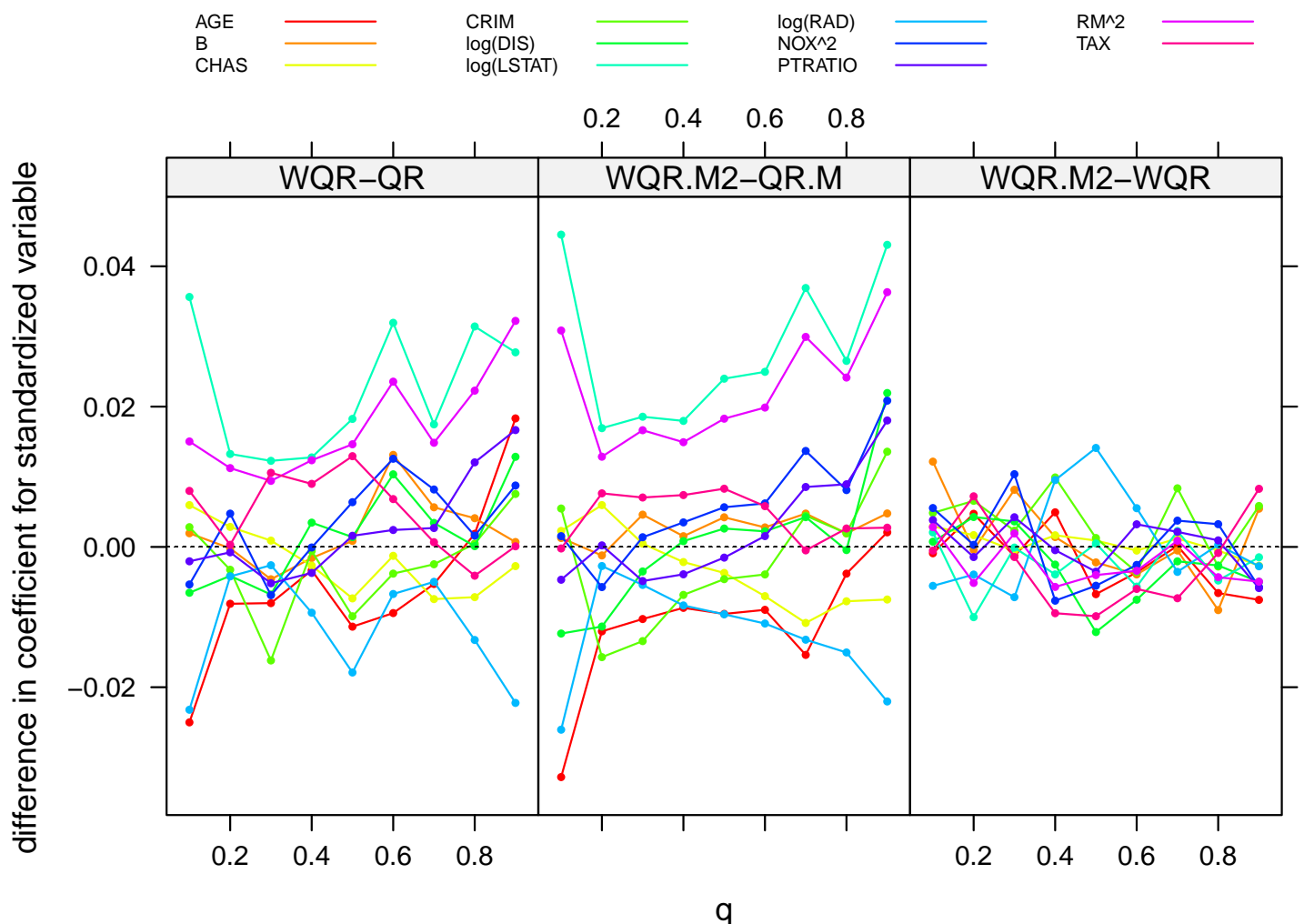

Figure 7: Differences in estimated coefficients of the standardized covariates for Boston Housing data at various quantiles $(q=0.1, \ldots, 0.9)$. 
presented here. We indicate a few promising directions.

The technique we have developed relies on a two-stage strategy, where the scale of the error distribution is estimated in a first stage, yielding scale factors used to estimate the quantile surface in the second stage. In our first scale-estimation scheme, an iterative algorithm can be used in the first stage, wherein scales are estimated, WQR.M is used to estimate the upper and lower quartile surfaces, with scales derived from these estimated surfaces. The second stage would then make use of these (more accurate) scales for final estimation of the quantile surfaces. In our second scaleestimation scheme, estimation of the median regression for the absolute residuals can be replaced by WQR.M estimation of the regression, again leading to more accurate estimates of the scales.

Throughout the simulations and data analysis, we have chosen to use a common scale estimate for estimation of the various quantile surfaces. This is in keeping with the notion of the errors following a scale family. Alternative approaches exist and would be appropriate when the assumption of a scale family is noticeably violated. Our methods can be focused directly on the scale near a particular quantile, say the upper quartile. For the first method, we can use, say, the difference between the estimated 90th and 60th percentile surfaces to obtain an estimate of the average density over this range of quantiles. With larger sample sizes, the band about the upper quartile can be shrunk, asymptotically focusing on the average density in a vanishingly small region about the upper quartile. The second method can also be focused on a quantile. Again considering a regression for the upper quartile, the two steps in the procedure can be altered as follows. First, fit the upper quartile surface to the data $\left(x_{i}, y_{i}\right)$ with QR (or QR.M). Extracting the residuals, $r_{i}$, from this fit provides an estimate of local deviations from the quartile surface. Second, run QR (or QR.M) with the absolute residuals as the response, using $\left(x_{i},\left|r_{i}\right|\right)$ as the data for the regression. The choice of quantile in this second regression determines how closely the scale estimate focuses on the density around the upper quartile. A regression for the 20th percentile extracts a scale estimate from one fifth of the distribution of the residuals; a regression for the 10th percentile extracts a scale estimate from one tenth of the distribution of residuals, and so on. Larger sample sizes support stable estimates of low percentile surfaces in this second regression, allowing us to better capture the densities near the upper quartile surface.

In this work, we have focused exclusively on linear quantile regression, but the proposed method can be readily extended to nonlinear quantile regression with heterogeneous errors. To do so, we need only modify the stage where the scale (as a function of covariates) is estimated. Following the path laid out here, we could estimate the upper and lower quartile surfaces through nonlinear quantile regression and obtain scale factors that vary locally, using these scales in the second stage. 


\section{Appendix}

Proof of Theorem 1.

$Z_{n}^{M}(\delta)=\sum_{i=1}^{n}\left\{\rho_{q}^{M}\left(w_{i}\left(u_{i}-x_{i}^{\top} \delta / \sqrt{n}\right)\right)-\rho_{q}\left(w_{i} u_{i}\right)\right\}$ can be decomposed into

$$
\sum_{i=1}^{n}\left\{\rho_{q}^{M}\left(w_{i}\left(u_{i}-x_{i}^{\top} \delta / \sqrt{n}\right)\right)-\rho_{q}\left(w_{i}\left(u_{i}-x_{i}^{\top} \delta / \sqrt{n}\right)\right)\right\}+Z_{n}(\delta) .
$$

Using similar arguments as in Lee et al. (2012) for consistency of modified quantile regression, we show that the first term becomes asymptotically negligible in determining the minimizer of $Z_{n}^{M}(\delta)$. First, we consider the expectation of the first term of the above decomposition.

$$
\begin{aligned}
& E\left(\sum_{i=1}^{n}\left\{\rho_{q}^{M}\left(w_{i}\left(u_{i}-x_{i}^{\top} \delta / \sqrt{n}\right)\right)-\rho_{q}\left(w_{i}\left(u_{i}-x_{i}^{\top} \delta / \sqrt{n}\right)\right)\right\}\right)+\frac{n q(1-q)}{2 \lambda_{\gamma}} \\
= & \sum_{i=1}^{n} \int_{x_{i}^{\top} \delta / \sqrt{n}}^{\frac{1-q}{\lambda \gamma w_{i}}+x_{i}^{\top} \delta / \sqrt{n}}\left(\frac{\lambda_{\gamma}}{2} \frac{q w_{i}^{2}}{1-q}\left(u-\frac{x_{i}^{\top} \delta}{\sqrt{n}}\right)^{2}-q w_{i}\left(u-\frac{x_{i}^{\top} \delta}{\sqrt{n}}\right)+\frac{q(1-q)}{2 \lambda_{\gamma}}\right) f_{i}\left(\xi_{i}+u\right) d u \\
+ & \sum_{i=1}^{n} \int_{-\frac{q}{\lambda_{\gamma} w_{i}}+x_{i}^{\top} \delta / \sqrt{n}}^{x_{i}^{\top} \delta / \sqrt{n}}\left(\frac{\lambda_{\gamma}}{2} \frac{(1-q) w_{i}^{2}}{q}\left(u-\frac{x_{i}^{\top} \delta}{\sqrt{n}}\right)^{2}-(q-1) w_{i}\left(u-\frac{x_{i}^{\top} \delta}{\sqrt{n}}\right)+\frac{q(1-q)}{2 \lambda_{\gamma}}\right) f_{i}\left(\xi_{i}+u\right) d u \\
= & \sum_{i=1}^{n} \int_{x_{i}^{\top} \delta / \sqrt{n}}^{\frac{1-q}{\lambda \gamma w_{i}}+x_{i}^{\top} \delta / \sqrt{n}} \frac{\lambda_{\gamma}}{2} \frac{q w_{i}^{2}}{1-q}\left(u-\frac{x_{i}^{\top} \delta}{\sqrt{n}}-\frac{1-q}{\lambda_{\gamma} w_{i}}\right)^{2} f_{i}\left(\xi_{i}+u\right) d u \\
+ & \sum_{i=1}^{n} \int_{-\frac{q}{x_{\gamma} w_{i}}+x_{i}^{\top} \delta / \sqrt{n}}^{x^{\top} \delta / \sqrt{n}} \frac{\lambda_{\gamma}}{2} \frac{(1-q) w_{i}^{2}}{q}\left(u-\frac{x_{i}^{\top} \delta}{\sqrt{n}}+\frac{q}{\lambda_{\gamma} w_{i}}\right)^{2} f_{i}\left(\xi_{i}+u\right) d u .
\end{aligned}
$$

Making use of a Taylor series expansion of $f_{i}$ at $\xi_{i}$ from (C-2), we can show that for $\lambda_{\gamma}=c n^{\alpha}$, the above expression is given by

$$
\frac{q(1-q)}{6 c^{2} n^{2 \alpha}} \sum_{i=1}^{n} \frac{f_{i}\left(\xi_{i}\right)}{w_{i}}+\frac{q(1-q)}{6 c^{2} n^{2 \alpha}} \sum_{i=1}^{n} \frac{f_{i}^{\prime}\left(\xi_{i}\right) x_{i}^{\top} \delta}{w_{i} \sqrt{n}}+o\left(n^{-2 \alpha+1 / 2}\right) .
$$

Note that $\sum_{i=1}^{n} f_{i}\left(\xi_{i}\right) / w_{i}=O(n)$ for $w_{i}=f_{i}\left(\xi_{i}\right)$, and $\sum_{i=1}^{n}\left\{f_{i}^{\prime}\left(\xi_{i}\right) / w_{i}\right\}\left(x_{i}^{\top} \delta / \sqrt{n}\right)=$ $O(\sqrt{n})$ as $f_{i}^{\prime}\left(\xi_{i}\right) / w_{i}, i=1, \ldots, n$ are uniformly bounded from the condition $(\mathrm{C}-2)$, and $(\mathrm{C}-1)$, and $\left|x_{i}^{\top} \delta\right| \leq\left\|x_{i}\right\|_{2}\|\delta\|_{2} \leq\left(\left\|x_{i}\right\|_{2}^{2}+\|\delta\|_{2}^{2}\right) / 2$ while $\sum_{i=1}^{n}\left\|x_{i}\right\|_{2}^{2}=O(n)$ from the condition (C-3). Thus, we have

$$
E \sum_{i=1}^{n}\left\{\left(\rho_{q}^{M}\left(w_{i}\left(u_{i}-x_{i}^{\top} \delta / \sqrt{n}\right)\right)-\left(\rho_{q}\left(w_{i}\left(u_{i}-x_{i}^{\top} \delta / \sqrt{n}\right)\right)\right\}-C_{n} \rightarrow 0 \quad \text { if } \alpha>1 / 4\right.\right.
$$


where $C_{n} \equiv-q(1-q) /\left(2 c n^{\alpha-1}\right)+q(1-q) /\left(6 c^{2} n^{2 \alpha}\right) \sum_{i=1}^{n} f_{i}\left(\xi_{i}\right) / w_{i}$. And similarly,

$$
\begin{aligned}
& \operatorname{Var}\left(\sum_{i=1}^{n}\left\{\rho_{q}^{M}\left(w_{i}\left(u_{i}-x_{i}^{\top} \delta / \sqrt{n}\right)\right)-\rho_{q}\left(w_{i}\left(u_{i}-x_{i}^{\top} \delta / \sqrt{n}\right)\right)\right\}\right) \\
= & \sum_{i=1}^{n} \frac{q^{2}(1-q)^{2} f_{i}\left(\xi_{i}\right)}{20 c^{3} n^{3 \alpha} w_{i}}+o\left(n^{-3 \alpha+1}\right) \rightarrow 0 \quad \text { for } \alpha>1 / 3
\end{aligned}
$$

Thus, under the condition that $\alpha>1 / 3$,

$Z_{n}^{M}(\delta)-Z_{n}(\delta)-C_{n}=\sum_{i=1}^{n}\left\{\rho_{q}^{M}\left(w_{i}\left(u_{i}-x_{i}^{\top} \delta / \sqrt{n}\right)\right)-\rho_{q}\left(w_{i}\left(u_{i}-x_{i}^{\top} \delta / \sqrt{n}\right)\right)\right\}-C_{n} \stackrel{p}{\rightarrow} 0$.

Finally, Theorem 5.1 of Koenker (2005) shows that $Z_{n}(\delta) \stackrel{d}{\rightarrow}-\delta \check{W}+\frac{1}{2} \delta^{\top} D_{2} \delta$ where $\check{W} \sim N\left(0, q(1-q) D_{2}\right)$, which completes the proof.

\section{Proof of Theorem 2.}

The proof of convergence is similar to the proof of Theorem 1, except that $u_{i}$ is now expressed as $\left(y_{i}-x_{i}^{\top} \beta\right) /\left(x_{i}^{\top} \tau\right)$. The behavior of $\sqrt{n}\left(\check{\beta}_{\tau}^{M}-\beta\right)$ follows from consideration of $\sum_{i=1}^{n}\left\{\rho_{q}^{M}\left(u_{i}-\frac{x_{i}^{\top} \delta}{x_{i}^{\top} \tau} \frac{1}{\sqrt{n}}\right)-\rho_{q}\left(u_{i}\right)\right\}$. First, we decompose the above expression.

$$
\begin{aligned}
Z_{n}^{M^{*}}(\delta) & =\sum_{i=1}^{n}\left\{\rho_{q}^{M}\left(u_{i}-\frac{x_{i}^{\top} \delta}{x_{i}^{\top} \tau} \frac{1}{\sqrt{n}}\right)-\rho_{q}\left(u_{i}\right)\right\} \\
& =\sum_{i=1}^{n}\left\{\rho_{q}^{M}\left(u_{i}-\frac{x_{i}^{\top} \delta}{x_{i}^{\top} \tau} \frac{1}{\sqrt{n}}\right)-\rho_{q}\left(u_{i}-\frac{x_{i}^{\top} \delta}{x_{i}^{\top} \tau} \frac{1}{\sqrt{n}}\right)\right\}+Z_{n}^{*}(\delta),
\end{aligned}
$$

where $Z_{n}^{*}(\delta)=\sum_{i=1}^{n}\left\{\rho_{q}\left(u_{i}-\frac{x_{i}^{\top} \delta}{x_{i}^{\top} \tau} \frac{1}{\sqrt{n}}\right)-\rho_{q}\left(u_{i}\right)\right\}$. Similar to the proof in Theorem 1 , for $\alpha>1 / 3$, it can be observed that $Z_{n}^{M *}(\delta)-Z_{n}^{*}(\delta)-C_{n}^{*} \stackrel{p}{\rightarrow} 0$, where

$$
\begin{aligned}
C_{n}^{*} & \equiv-q(1-q) /\left(2 c n^{\alpha-1}\right)+q(1-q) /\left(6 c^{2} n^{2 \alpha}\right) \sum_{i=1}^{n} f_{i}\left(\xi_{i}\right)\left(x_{i}^{\top} \tau\right) \\
& =-q(1-q) /\left(2 c n^{\alpha-1}\right)+q(1-q) /\left(6 c^{2} n^{2 \alpha}\right)\left(n \cdot f_{u}(0)\right) .
\end{aligned}
$$

Thus, asymptotic behavior of $\check{\beta}_{\tau}^{M}$ is equivalent to that of $\check{\beta}_{\tau}$. Koenker and Zhao (1994) show that $\sqrt{n}\left(\check{\beta}_{\tau}-\beta\right) \stackrel{d}{\rightarrow} N\left(0, \frac{q(1-q)}{f_{u}^{2}(0)} D_{2}^{*-1}\right)$. Now, $\check{\beta}_{\hat{\tau}}$ with a $\sqrt{n}$-consistent estimator of $\tau$ up to scale will have the same asymptotic behavior as $\check{\beta}_{\tau}$ as shown in Theorem 2.1 of Koenker and Zhao (1994). 


\section{References}

Bassett, G. and Koenker, R. (1978). Asymptotic theory of least absolute error regression, Journal of the American Statistical Association 73(363): 618-622.

Gilley, O. and Pace, R. K. (1996). On the harrison and rubinfeld data, Journal of Environmental Economics and Management 31: 403-405.

Gutenbrunner, C. and Jurečková, J. (1992). Regression rank scores and regression quantiles, The Annals of Statistics 20(1): 305-330.

He, X. (1997). Quantile curves without crossing, The American Statistician 51(2): 186-192.

Horowitz, J. (1998). Bootstrap methods for median regression models, Econometrica 66: $1327-1352$.

Jung, Y. (2010). Regularization of Case Specific Parameters: A New Approach for Improving Robustness and/or Efficiency of Statistical Methods, PhD thesis, The Ohio State University.

Jung, Y., MacEachern, S. N. and Lee, Y. (2010). Window width selection for $l_{2}$ adjusted quantile regression, Technical Report 835, Department of Statistics, The Ohio State University.

Koenker, R. (2005). Quantile Regression, Cambridge University Press.

Koenker, R. and Bassett, G. (1978). Regression quantiles, Econometrica 46(1): 33-50.

Koenker, R. and Machado, J. A. F. (1999). Goodness of fit and related inference processes for quantile regression, Journal of the American Statistical Association 94(488): 1296-1310.

Koenker, R. and Park, B. J. (1996). An interior point algorithm for nonlinear quantile regression, Journal of Econometrics 71: 265-283.

Koenker, R. W. and D'Orey, V. (1987). Algorithm as 229: Computing regression quantiles, Journal of the Royal Statistical Society. Series C (Applied Statistics) 36(3): 383-393.

Koenker, R. and Zhao, Q. (1994). L-estimation for linear heteroscedastic models, Journal of Nonparametric Statistics 3(3): 223-235.

Lee, Y., MacEachern, S. N. and Jung, Y. (2012). Regularization of case-specific parameters for robustness and efficiency, Statistical Science 27(3): 350-372. 
Nychka, D., Gray, G., Haaland, P., Martin, D. and O'Connell, M. (1995). A nonparametric regression approach to syringe grading for quality improvement, Journal of the American Statistical Association 90(432): 1171-1178.

Rubinfeld, D. H. D. L. (1978). Hedonic housing prices and the demand for clean air, Journal of Environmental Economics and Management 5: 81-102. 